\title{
COVID-19 Reducing the Risks: Telemedicine is the New Norm for Surgical Consultations and Communications
}

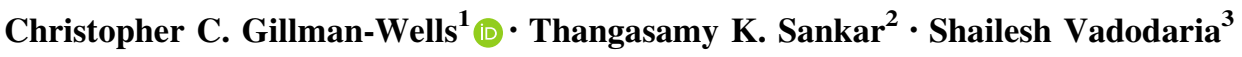

Received: 21 June 2020/Accepted: 27 July 2020/Published online: 3 September 2020

(C) Springer Science+Business Media, LLC, part of Springer Nature and International Society of Aesthetic Plastic Surgery 2020

\begin{abstract}
Introduction COVID-19, a worldwide pandemic, has enforced a national lockdown in the UK which produced a paradigm shift about the way medical practitioners would perform consultations and communication with their patients. Senior authors realised that in lockdown there was only one option to see a patient: virtual consultation via telecommunication technologies. This paper will discuss the current benefits and considerations of Telemedicine, particularly in plastic surgery, to decipher the next route of action to further validate its use for future implementation. Method A detailed literature review was carried out comparing papers from 1992 to 2020. A survey of 122 consultant plastic surgeons found an encouraging result as $70 \%$ positively embraced the suggestion of Telemedicine in their current practice.

Discussion Telemedicine produced equal or improved patient satisfaction. Its utilisation reduced cost for patient, clinic and consultant. With accessibility to a large percentage of the population, Telemedicine enables infection control and adherence to social distancing during COVID19. Considerations include dependability on internet access, legal aspects, cyber security and General Data Protection Regulation (GDPR), the inability to perform
\end{abstract}

Christopher C. Gillman-Wells

gillmanwellsc@icloud.com

1 University of Leeds Medical School - Year One, 51 St. Nicholas Crescent, Pyrford GU228TD, UK

2 Kettering General Hospital NHS Foundation Trust, Kettering, UK

3 Clementine Churchill BMI Hospital, London Harley Health Village, London MACS Clinic, Watford, London, UK palpation or physical inspection and psychological impacts on the patient.

Conclusion In modern times, Telemedicine has become more accessible and COVID-19 has made it more applicable than ever before. More in-depth research is needed for validation of this technique within plastic surgery. While maintaining quality of care and a vital role in social distancing, there is a strong need for standardisation of Telemedicine processes, platforms, encryption and data storage.

Level of Evidence $V$ This journal requires that authors assign a level of evidence to each article. For a full description of these Evidence-Based Medicine ratings, please refer to the Table of Contents or the online Instructions to Authors www.springer.com/00266.

Keywords Postoperative $\cdot$ Telemedicine $\cdot$ COVID-19

\section{Introduction}

Telemedicine is defined as the use of telecommunication and online technologies for the provision of medical services or care [1]. It can be used for transfer and analysis of postoperative wound images, X-rays, over-the-phone appointments and real-time video consultations, all from a distance. Since the early twentieth century, when Telemedicine was introduced, technology has evolved exponentially providing the opportunity for more efficient and effective healthcare [2]. Telemedicine is a rapidly advancing service which provides a platform for education and training, a faster diagnosis of health-related issues, reduced cost for both healthcare services and patients, as well as protection from public infection risks. In the past twenty years, there have been five pandemic outbreaks: 
SARS (2003), Swine Flu (2009), Ebola (2014), ZIKA (2015) and now COVD-19 [3]. The first four outbreaks mentioned have had a total death toll of about 210,000 [4].

COVID-19 has been the cause of nearly 400,000 deaths from December 31st 2019 to 7th June 2020 [5]. Its high infection and mortality rate have forced a worldwide lockdown which significantly reduced the possibility of face-to-face consultation and the need for Telemedicine has become a must. Implementation of virtual consulting has the potential to limit the spread of disease providing safety from current and future outbreaks for both healthcare services and the patient. As we become more reliant on Telemedicine, extensive research must be carried out to provide evidence of its benefits but also the real and possible downsides. The General Medical Council (GMC) states that the two key issues with using Telemedicine are consent and continuity of care and that whichever software platform is employed for the virtual consultation, it does not affect the physicians ability to follow the law and their guidance [6].

Telemedicine has been investigated in abundance for its use in postoperative care and has found to produce excellent clinical outcomes, time and cost-saving benefits as well as improved patient satisfaction [7]. Far less research has been carried out on pre-surgical consultations; an area where future studies should aim to target to fully gain sufficient reliability for the employment of Telemedicine in place of traditional face-to-face consultations.

The aim of this paper is to discuss what we already know about Telemedicine, how it can be used in today's climate, particularly in plastic surgery, and to decipher the next route of action to further validate its use for future implementation.

\section{Virtual Consulting in Surgery}

Virtual consulting provides the opportunity for use in both pre-surgery consultations and postoperative care. There are currently far fewer preoperative studies, particularly for aesthetic plastic and reconstructive surgery, than those investigating postoperative care. Furthermore, each study is aimed at a completely different field of medical care or treatment including maxillofacial surgery [8], management of pheochromocytoma [9] and laparoscopic surgeries [10]. This may be seen as an advantage to prove its use in a variety of healthcare surgery types but also an issue of reliability as to whether similar results can be reproduced if the individual investigations were to be repeated. Despite this, these results have provided the same or increased patient satisfaction with the use of real-time video over traditional consultations. While Telemedicine still remains in early stages of development, it has been shown to aid paediatric surgeons with increased access to care in the preoperative setting but also a number of barriers to its adoption [11]. These include an inherent desire to see a physician in person, regulatory barriers of teleconsultation services and a plethora of legal considerations including compliance requirements with GDPR and insurance companies particularly using Telemedicine in private practice.

Rapidly advancing technology has allowed successful identification of postoperative complications. This enables same or next day replies from physicians, all from a distance [12]. With an increasing amount of patients wanting to converse more frequently with their physician, Telemedicine enables patient-physician contact at virtually any time regardless of location, provided you have access to internet. JP Gunter, a senior plastic surgeon, has employed the use of virtual consulting since 2002. From his work, he experienced only $5 \%$ of patients who sent inadequate images of the treatment area. In response, rapid follow-up information is sent directly to the patient about how to take the photographs correctly [13]. This is supported by TA Sayed who wrote that Telemedicine allowed remote monitoring to facilitate wellness of patients in postoperative care and maintained the same level of efficacy as a face-toface consultation [14]. When undergoing these online consultations software such as Zoom and Microsoft Teams allow screen sharing options which unlocks a multitude of uses. Screen sharing allows the surgeon to show detailed presentations and diagrams regarding the requested surgery, and techniques such as morphing of images to discuss the expected outcome in aesthetic plastic surgery treatments.

A study by Murphy et al. [15] assessed the use of postoperative images sent by patients for wound healing, compared to face-to-face postoperative consultations. No difference was experienced in the quality of care between the two methods. This provided evidence of accurate and efficient care from a distance. It is important to note that the high degree of confidence with this technique, according to this study, was that participants were trained specially to use the technology and acquired software to provide a higher quality of images for analysis. This shows that to get the results we need to become reliant upon Telemedicine. There will be an additional cost to develop Telemedicine systems with encryption and data storage. It needs integration with current systems for seamless provision of medical care. The Telemedicine usage needs to be validated. These developments will need finance and human resources. Before we have widespread adoption of Telemedicine, we need to appraise its cost-benefit ratio.

It is common for patients to worry about their wounds postoperation. Having the ability to quickly take photographs and send them via text or email may be able to alleviate this worry. Telemedicine enables the clinician to assure patients of the level of urgency required and whether 
travel to the hospital is actually needed. One study found that $99 \%$ of patients would use Telemedicine again as a method for postoperative care [16]. Of those using Telemedicine, no postsurgical complications were missed. It is possible that complications were missed such as septicaemia causing a delayed effect and sepsis risk. None were documented here. This study also emphasises the point that in low-risk surgeries there are multiple techniques in which to seal an incision which do not require follow-up in-clinic visit. Moreover, if a patient is still worried about their wound recovery, they still have the ability to book and go into hospital for a traditional check-up. Multiple studies confirm the effectiveness of using Telemedicine in postoperative care [17-19].

No studies have been found that provide evidence against the use of Telemedicine or that it increased the risk of complications of postoperative care. What should be considered when moving forward is that the majority of research so far has been aimed at postoperative care. Future studies should target more preoperative use of Telemedicine to gain sufficient and reliable evidence of its benefits. Another route for future studies would be to investigate pre-surgery and postoperative care of treatments associated with a higher risk of complications, as most reviewed here are associated with low-risk complications. This would provide a more intensive follow-up of complex cases. If future evidence is produced showing no loss in patient satisfaction and quality of care in preoperative consultations and surgeries that are at higher risk of complications, then it provides us with the opportunity to replace the traditional in-clinic visits with real-time video consultations. This saves time for the patient and physician as well as greatly reducing overall cost of healthcare.

\section{Senior Author Survey}

A recent online survey using Smart Survey software amongst 122 consultant aesthetic plastic surgeons revealed a very significant consensus for the use of virtual consulting. The survey provided validation of views surrounding the use of Telemedicine, especially in this current climate as $64 \%$ reported they would use the virtual platform for pre- and postsurgical consultations. The senior author (SV) has used virtual consultations for the last 7 years for pre- and postoperative care and increased its use extensively during the current lockdown in the UK while maintaining the highest patient satisfaction rate. From the last 97 virtual consultations, we have found $99 \%$ high satisfaction rate from the quality of virtual consultation and the exchange of information.

\section{Reduced Infection Risk with Time and Cost Benefits}

Evidence has been provided to show that patient satisfaction and quality of care can be upheld using Telemedicine technology. An added incentive supporting the use of Telemedicine is the advantage of time saving and costeffective benefits. Reducing travel also has the environmental advantage of reducing $\mathrm{CO}_{2}$ emissions. Employing the use of Telemedicine in Minneapolis found a substantial reduction in travel cost, as well as reducing the chance of reduced income from missing days of work due to traditional face-to-face visits. With this in mind, patients may need to take time off work regardless of the video consultation length as it depends on when during the day both participants can agree a time. Patients also need time to reflect on the consultation. Each patient in this study had an average $\$ 50$ saving using virtual consulting with no postoperation complication being missed. This highlights the crucial point that quality of care can be upheld as well as the added benefits [16]. This is supported by Viers et al. [19] and Westra and Niessen [20] who found equal patient satisfaction between the traditional consultations and video consultations while granting both time and cost-saving benefits. Employing the use of skype calls for postoperative care was found to increase patient satisfaction compared to the traditional group. Video calls only lasted an average of $2.71 \mathrm{~min}$ for each consultation. People in the non-Telemedicine group found that traditional consultations did not provide enough interaction and thus provided a reduced quality of care and satisfaction [21]. Time and cost benefits are largely affected by distance from the hospital or clinic. It is assumed that the more isolated the patient is from the physician the more likely they are to use virtual consulting. Canon et al. [22] found that every 23 miles a patient resides from their particular clinic, patients are $111 \%$ more likely to use Telemedicine. What adds to this time-saving benefit of Telemedicine is the removal of time spent doing nonclinical-related activates such as checking in/out and transfer from waiting rooms to examination rooms [23]. Moreover, these time reductions improve availability to high demand treatments and specialities which can now be accessed from remote sites.

During a global pandemic, Telemedicine provides us with the opportunity to reduce chances of infections that may occur travelling to or from hospitals or directly from the hospital itself [24]. There are no guidelines for preoperative evaluations but it is suggested that video consultations can replace traditional consultations during COVID19 , which can include using a local primary care physician to carry out a physical examination, if needed, on the patient [25]. Postoperatively, there may be some patients which require removal of bandages or stitches but despite this inevitability, using video calls for all other follow-ups 
inhibits exposure to infection and helps to adhere to social distancing.

\section{Unique Use of Smart Phones}

With the modern advancement of smart-phone technology, Telemedicine is now easier and more accessible than ever. Studies have shown that those from low-income backgrounds and people of older age still have relatively high rates of smart-phone ownership [26, 27]. Diagnoses can be correctly identified from a picture sent from a smart phone. It is easy to be able to produce high-quality images with minimal instruction. One study used a Nokia 6600 to take images and transfer them via email. Out of 96 patients, all postoperative complications were assessed successfully by 3 physicians, with only one of those patient's needing to return to hospital [28]. Using this technique avoided any unnecessary trips to the healthcare centre from patients, saving both the physicians and the patients time and money, including removal of risk of exposure to infection. Patients have been able to send images to physicians 48-72 $\mathrm{h}$ following surgery which enables early detection of complications and next day treatment, if needed. Improved quality of care has been reported in $96 \%$ of patient's [27]. In one study, relatively few issues were identified such as older patient's struggling with technology, obese patient difficulties with taking photographs or challenging areas such as groins; however, $80 \%$ of images provided were sufficient for diagnostic purposes [29].

\section{Telemedicine Considerations}

So far, the benefits and potential for improved quality of care and patient satisfaction associated with the use of Telemedicine have been discussed. There are some considerations to take note of. Sayed TA, MD, has warned that the use of Telemedicine in the preoperative setting may eliminate the patient from candidacy for a desired treatment. It is possible for a photograph to be misdiagnosed and in-person examinations may alter a physician's recommendations. This highlights the worry of virtual consultations limiting the amount of information that can be exchanged and inability to perform effective diagnosis [30]. Connectivity provides a barrier to the use of Telemedicine. Low bandwidth produces low-quality images or poor real-time video quality. Purchases and set-up of HD camera or improved software may be time-consuming or require training of staff. There is also the risk that using online software instead of meeting face-to-face may be detrimental to the patient-doctor relationship. Giving patients the ability to have direct contact with the physician potentially allows them to start contacting their physician whenever they desire, unnecessarily. This may distract the physician from other patient's which may cause resentment or reduced quality of care. Inadequate language skills provide a barrier to Telemedicine. If a patient or physician struggles to communicate effectively, it is easier to find ways around this in person with other communication techniques. On the other hand, most software's that are used for virtual consulting provide side bars for written communication or ability to screen share to allow easier transfer of information. The majority of PCs, laptops and smart phones consist of good-quality cameras in order to use Telemedicine. The availability of internet with consistently good bandwidth is improving day by day.

Psychological impact plays an important role in face-toface or virtual consulting. If we are to use this technology, then it is crucial to be able to maintain trust and a good patient-doctor relationship. There are nine forms of nonverbal communication which are important for the development and maintenance of this relationship [31]. With the exception of touch and physical closeness, the other seven of these forms are provided for using Telemedicine [32]. On the other hand, Lemos Da Luz explains that $80 \%$ of communication is non-verbal, such as hand gestures, posture and eye contact and that it is difficult to determine whether online images can substitute for personal contact [33]. This emphasises an important point that Telemedicine, due to its novelty, has its limitations which only face-to-face consultations can accommodate for. Palpation is required for certain procedures which can only be carried out by the surgeon during traditional consultations and 3D inspection is extremely challenging to be completed accurately without in-person contact. A summary table has been placed in Appendix listing the benefits and considerations of Telemedicine.

Other questions that should be addressed in the future are: How responsibility of the patient is determined throughout the process of using Telemedicine, is it always the surgeon or shared by accompanying nurses and physicians? How do we handle the difficult patient and what are the psychological/legal complications of using Telemedicine for breast, abdomen and external genitalia surgeries? Can Telemedicine ever be truly safe from cyberattacks or loss of personal data?

\section{Legal Considerations of Telemedicine}

Using electronic devices to send any information regardless of what it is associated with has the potential to be leaked or hacked. There has been increased concern over privacy of sending medical information [34]. For safety, security and confidentiality purposes, there is a strong need for verified authentication, encryption of data, security polices and detailed contracts providing all the information necessary regarding the use of telemedicine as a service 
[35]. The vast majority of healthcare providers or hospitals have accessibility to such protection software but this is not always guaranteed from the side of the patient. One simple solution to this is to send online data that can only be accessed or opened via a password-protected encryption, known only to those who are involved in the specific treatment. Patient data can also be anonymised to reduce risk of breaking confidentiality. An example of this would be to blur out areas of non-interest. In plastic surgery, if a patient where to have a rhinoplasty, areas not affected by the surgery may be blurred or covered to hide the identity of the patient [36].

Access to folders that contain video logs should also be password protected. Many third-party companies can provide secure encryption that are safe, such as Azure or AWS $[37,38]$. There are many more external small suppliers that can provide encryption services for online data.

When recording video consultations, it is imperative patients are fully informed to who has access to the data they are sending online. This includes the potential use of video $\log$ s and images for training or educational purposes, this must be agreed to via online recording and or by consent form before any Telemedicine-associated treatment can begin. Furthermore, recorded consultations can be used as legal evidence if any issues arise between the patient and physician. Due to this, recordings of consultations have the ability to reduce misconduct from both patient and clinician. It is advised by the GMC that when providing services remotely you work within your competence, discuss the use of Telemedicine with your responsible officer at appraisal and check you have adequate indemnity cover for virtual consultations [6]. The ICO recognises that during this extraordinary time organisations are having to adapt to provide vital healthcare and other services. It is stated that tools presented to them which have the capacity to recover or deal with this crisis will be fast tracked for support [39]. The use of Telemedicine needs to be compliant with GDPR rules and regulations for data protection.

\section{Conclusion}

Telemedicine has existed for a long time but modern technologies have made it more accessible, and COVID-19 has made it more applicable than ever before. Evidence has been provided to show that the use of Telemedicine maintains or improves patient satisfaction and quality of care. This novel method of consultation should be supported by governments of various countries and regulatory bodies, the WHO and national and international associations of subspecialties due its inherent time/cost-saving element beneficial to both patient and healthcare provider, with the added benefit of reducing infection risk and its major role in social distancing. Telemedicine is not perfect and with few current studies associated with plastic and aesthetic surgery, there are real and important considerations to resolve before we can evolve into a new era of a virtual rather than face-to-face consultations. With global support to aid to the validation process, we should be able to employ a virtual method of consultation without compromising quality of care for our patients.

Currently, there are many Telemedicine communication platforms available with variable features, security and storage capacity. To allow substantial use of Telemedicine, there is an essential need for standardisation of virtual consultation processes, platforms, encryption and data storage.

Acknowledgements We are very grateful for Prof. James Frame's invaluable guidance and suggestions towards this publication.

\section{Compliance with Ethical Standards}

Conflict of interest The authors declare that they have no conflicts of interest to disclose.

Ethical Approval This article does not contain any studies with human participants or animals performed by any of the authors. For this type of study, informed consent is not required.

Informed Consent For this type of study, informed consent is not required.

\section{Appendix}

A table to summarise the benefits and considerations discussed in this paper associated with the implementation of Telemedicine for virtual consultation.

\begin{tabular}{|c|c|}
\hline Benefits of telemedicine & Considerations of telemedicine \\
\hline $\begin{array}{l}\text { Equal/improved patient } \\
\text { satisfaction and quality of care }\end{array}$ & $\begin{array}{l}\text { Legal aspects (e.g. consent, } \\
\text { confidentiality) }\end{array}$ \\
\hline $\begin{array}{l}\text { Reduced cost for patient, hospital } \\
\text { clinic and consultant }\end{array}$ & $\begin{array}{l}\text { Only usable with internet } \\
\text { connection (dependant on } \\
\text { bandwidth) }\end{array}$ \\
\hline $\begin{array}{l}\text { Time effective/No travel } \\
\text { (environmentally friendly) }\end{array}$ & $\begin{array}{l}\text { Psychological impact on the } \\
\text { patient and potential effect on } \\
\text { patient-doctor relationship }\end{array}$ \\
\hline $\begin{array}{l}\text { Infection control-adherence } \\
\text { with social distancing policy in } \\
\text { the current climate }\end{array}$ & $\begin{array}{l}\text { Cyber security issues and patient } \\
\text { safety (GDPR rules and } \\
\text { regulations) }\end{array}$ \\
\hline $\begin{array}{l}\text { Accessible to a large percentage } \\
\text { of the population }\end{array}$ & $\begin{array}{l}\text { Unable to perform palpation and } \\
\text { physical inspection }\end{array}$ \\
\hline $\begin{array}{l}\text { Easy availability of user-friendly } \\
\text { multiple devices (e.g. smart- } \\
\text { phone use, laptops, tablets) }\end{array}$ & $\begin{array}{l}\text { Essential requirement of } \\
\text { standardisation of Telemedicine } \\
\text { processes, platforms, } \\
\text { encryption and data storage }\end{array}$ \\
\hline
\end{tabular}




\section{References}

1. Zhang X-Y, Zhang P (2016) Telemedicine in clinical setting. Exp Ther Med 12(4):2405-2407

2. Asiri A, AlBishi S, AlMadani W et al (2018) The use of telemedicine in surgical care: a systematic review. Acta Inform Med 26(3):201-206

3. Huremovic D (2019) Brief history of pandemics (pandemics throughout history). Psychiatry Pandemics 16:7-35

4. LePan N (2020) Visualising the history of pandemics. Visual Capitalist. https://www.visualcapitalist.com/history-ofpandemics-deadliest/. Accessed 8 June 2020

5. European Centre for Disease Prevention and Control (2020) COVID-19 situation update worldwide, as of 7 June 2020. https:// www.ecdc.europa.eu/en/geographical-distribution-2019-ncovcases. Accessed 13 June 2020

6. Remote Consultations (2020) General medical council. https:// www.gmc-uk.org/ethical-guidance/ethical-hub/remoteconsultations. Accessed 13 June 2020

7. Williams AM, Bhatti UF, Alam HB et al (2018) The role of telemedicine in postoperative care. Mhealth 4:11

8. Rollert MK, Strauss RA, Abubaker AO et al (1999) Telemedicine consultations in oral and maxillofacial surgery. J Oral Maxillofac Surg 57(2):136-138

9. Heslin MJ, Liles J-S, Moctezuma-Velaquez P (2019) The use of telemedicine in the preoperative management of pheochromocytoma saves resources. Mhealth 5:7

10. Lee S, Broderick TJ, Haynes J et al (2003) The role of lowbandwidth telemedicine in surgical pre-screening. J Pediatr Surg 38(9):1281-1283

11. Lesher AP, Shah SR (2018) Telemedicine in the Preoperative Experience. Semin Pediatr Surg 27(2):102-106

12. Shaw S, Wherton J, Vijayaraghavan S, et al (2018) Advantages and limitations of virtual online consultations in a NHS acute trust: the VOCAL mixed-methods study. Health Serv Deliv Res 6(21)

13. Examining Virtual Consulting (2020) Plastic surgery practice. https://www.plasticsurgerypractice.com/practice-management/ examining-virtual-consulting/. Accessed 4 June 2020

14. Sayed TA (2020) The future of telemedicine in aesthetics. Practical dermatology. https://practicaldermatology.com/articles/ 2015-dec/the-future-of-telemedicine-in-aesthetics. Accessed 4 June 2020

15. Murphy RX, Bain MA, Wasser TE et al (2006) The reliability of digital imaging in the remote assessment of wounds: defining a standard. Ann Plast Surg 56(4):431-436

16. Dirnberger J, Waisbren S (2020) Efficacy of telehealth visits for postoperative care at the Minneapolis VA. Am J Surg S0002-9610(20):30016-30017

17. Hwa K, Wren SM (2013) Telehealth follow-up in lieu of postoperative clinic visit for ambulatory surgery: results of pilot program. JAMA Surg. 148(9):823-827

18. Broman KK, Roumie CL, Stewart MK et al (2016) Implementation of telephone postoperative clinic in an integrated health system. J Am Coll Surg 223(4):644-651

19. Viers BR, Lightner DJ, Rivera ME et al (2015) Efficiency, satisfaction, and costs for remotes video visits following radical prosatectomy: a randomised controlled trial. Eur Urol 68(4):729-735

20. Westra I, Niessen FB (2015) Implementing real time video consultation in plastic surgery. Aesthetic Plast Sur. 39(5):783-790
21. Sharareh B, Schwarzkopf R (2014) Effectiveness of telemedical applications in postoperative follow-up after total joint arthroplasty. J Arthroplasty 29(5):918-922

22. Canon S, Shera A, Patel A et al (2014) A pilot study of telemedicine for post-operative urological care in children. J Telemed Telecare 20(8):427-430

23. Ellimoottil C, Boxer RJ (2018) Bringing surgical care to the home through video visits. JAMA Surg 153(2):177-178

24. Hau YS, Kim JK, Hur J et al (2020) How about actively using telemedicine during the COVID-19 pandemic? J Med Syst 44(6): 108

25. Hakim AA, Kellish AS (2020) Implications for the use of telehealth in surgical patinets during the COVID-19 pandemic. Am J Surg

26. Vangeepuram N, Mayer V, Fei K et al (2018) Smartphone ownership and perspectives on health apps among a vulnerable population in East Harlem, New York. Mhealth 4:31

27. Pozza DE, D’Souza GF, DeLeonibus A et al (2017) Patient satisfaction with an early smartphone-based cosmetic surgery postoperative follow-up. Aesthet Surg J. 38(1):101-109

28. Martinez-Ramos C, Cerdan MT, Lopez RS (2009) Mobile phonebased telemedicine system for the home follow-up of patients undergoing ambulatory sugery. Telemed J E Health 15(6):531-537

29. Gunter R, Fernandes-Taylor S, Mahnke A et al (2016) Evaluating patient usability of an image-based mobile health platform for postoperative wound monitoring. JMIR Mhealth Uhealth 4(3):113

30. Chada BV (2017) Virtual consultations in general practice: embracing innovation, carefully. Br J Gen Pract 67(659):264

31. Grzybowski SC, Stewart MA, Weston WW (1992) Nonverbal communication and the therapeutic relationship: leading to a better understanding of healing. Can Fam Phys 38:1994-1998

32. Toh N, Pawlovich J, Grzybowski S (2016) Telehealth and patinet-doctor relationships in rural and remote communities. Can Fam Phys 62(12):961-963

33. Lemos da Luz P (2019) Telemedicine and the doctor/patient relationship. Arq Bras Cardiol 113(1):100-102

34. Caryl C (1997) Malpractice and other legal issues preventing the development of telemedicine. J Law Health. 12(1):173-204

35. Stanberry B (2006) Legal and ethical aspects of telemedicine. J Telemed Telecare 12(4):166-175

36. Gardiner S, Hartzell T (2011) Telemedicine and plastic surgery: a review of its applications, limitations and legal pitfalls. JPRAS 65(3):47-53

37. Shanahan HP, Owen AM, Harrison AP et al (2014) Bioinformatics on the cloud computing platform azure. PLoS ONE 9(7):102642

38. Griebel L, Prokosch H-U, Kopcke F et al (2015) A scoping review of cloud computing in healthcare. BMC Med Inform Decis Mak 15:17

39. The ICO's regulatory approach during the coronavirus public health emergency (2020) Information Commissioner's Office. https://ico.org.uk/media/about-the-ico/policies-and-procedures/ 2617613/ico-regulatory-approach-during-coronavirus.pdf. Accessed 13 June 2020

Publisher's Note Springer Nature remains neutral with regard to jurisdictional claims in published maps and institutional affiliations. 